Received 00th January 20xx, Accepted 00th January 20xx DOI: $10.1039 / \times 0 \times x 00000 x$

\title{
Irreproducibility in the triboelectric charging of insulators: evidence of a non-monotonic charge versus contact time relationship
}

\author{
Jinyang Zhang, ${ }^{\text {a }}$ Chao Su, ${ }^{\mathrm{b}}$ Fergus J. M. Rogers, ${ }^{\mathrm{c}}$ Nadim Darwish, ${ }^{\mathrm{a}}$ Michelle L. Coote, ${ }^{* \mathrm{c}}$ and Simone \\ Ciampi*a
}

\begin{abstract}
Here, we investigate the development and relaxation of static charges on the surface of plastic materials that are first brought in contact, and then macroscopically separated. Experimentalists dealing with the static electrification of insulators are aware of difficulties predictably attaining, and precisely reproducing, a given charging magnitude. Here we have observed for the first time that in homo-systems (e.g. PTFE rubbed against PTFE) charge densities reach the maximum value after a material-specific contact time. Attempts to charge a sample beyond its peak value leads to a progressive drop in charge. We propose this drop to result both from the electrostatically driven segregation of polymer ionic fragments, as well as from the discharge of unstable fragments by dielectric breakdown when a sufficiently high surface charge density is reached. We therefore highlight the general existence of two branches in the charging versus charging time curve: the assumption of a monotonous charging slope holds only left or right of the charging maxima and to achieve a specific charge density care has to be taken to remain within one branch. Differences between materials in the tribocharging peak time are shown to reflect difference in material transfer rates and water adsorption, rather than differences in electronic factors such as the relative stability of cationic and anionic fragments.
\end{abstract}

\section{Introduction}

Triboelectricity is the phenomena by which dielectric materials gain a net electric charge upon contact and separation with other insulators, ${ }^{1-4}$ semiconductors ${ }^{5}$ or metals. ${ }^{6-8}$ The study of triboelectricity has strong roots in the 1970 s physics-dominated research on xerography, but there are several more recent examples of processes harnessing the electrification of insulators, such as electrostatic separation of biomass, ${ }^{9}$ glass panel cleaning in solar farms, ${ }^{10}$ and the recovery of alternating current from the dissipation of friction in triboelectric nanogenerators (TENGs). ${ }^{11-17}$ Through Kelvin probe force microscopy (KPFM) ${ }^{18-20}$ and Faraday pail measurements, ${ }^{21}$ static charges present on a surface can be accurately mapped and measured. A number of studies have used these types of

a. School of Molecular and Life Sciences, Curtin Institute of Functional Molecules and Interfaces, Curtin University, Bentley, Western Australia 6102, Australia.

b. School of Energy and Power Engineering, Jiangsu University of Science and Technology, Zhenjiang 212003, P.R. China.

c. ARC Centre of Excellence for Electromaterials Science, Research School of Chemistry, Australian National University, Canberra, Australian Capital Territory 2601, Australia.

+ Electronic Supplementary Information (ESI) available: [details of any supplementary information available should be included here]. See DOI: $10.1039 / x 0 x x 00000 x$ measurements to highlight the complexity of the triboelectrification process - ionization energy, electron affinity, 22, 23 moisture, ${ }^{24,} 25$ adhesion, ${ }^{5}$ hardness, ${ }^{5,} 26$ contact force, ${ }^{19}$ gas dissolution, ${ }^{27}$ as well as the exact surface chemistry $^{23}$ - all play a role in it. A correct and complete understanding of the process is still lacking. A significant amount of material is transferred upon contact, ${ }^{5,18}$ and it thus follows that the study of contact charging on insulators will be more easily studied in a single-material model system. Surprisingly, the study of contact electrification has so far mainly focused on dynamic contacts between different materials, ${ }^{28-30}$ and reports on the contact and charging between identical materials are far less frequent. ${ }^{26}$

Herein, by studying through Faraday pail and atomic force microscopy (AFM) the contact electrification of systems comprising of a single material, we reveal that the build-up of electrical charges does not increase monotonically with the duration of the charging step: charging magnitude peaks at intermediate times and then drops. There is limited published data on prolonged tribocharging procedures, but our findings are in contrast with a monotonic charge growth reported for stainless steel spheres rolled over polystyrene. ${ }^{31}$ An explanation based on the simultaneous role of adsorbed water, material transfer and electrostatic-driven segregation of charged polymer fragments is proposed. The use of a single-material 


\section{ARTICLE}
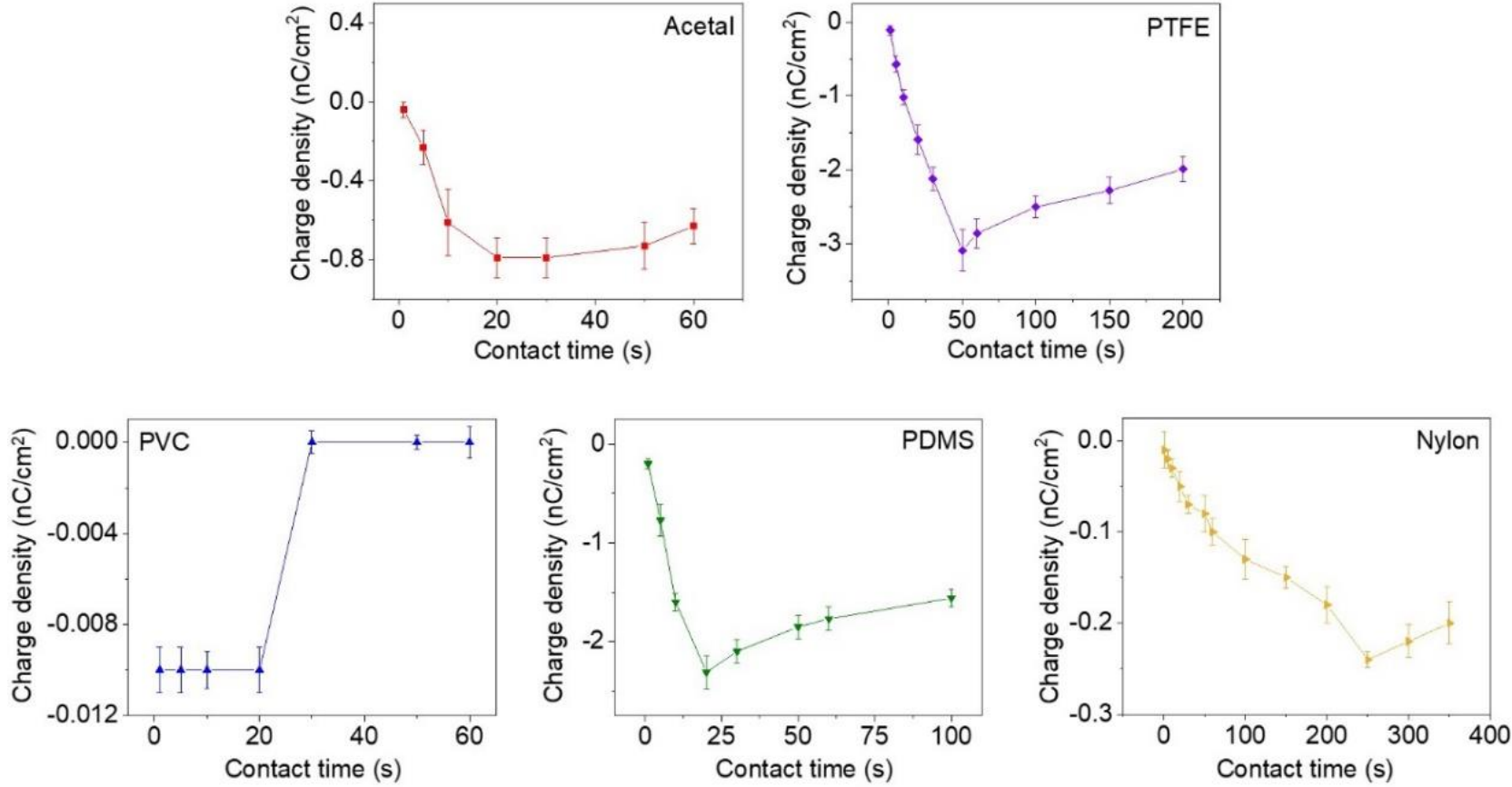

Fig. 1. Triboelectrification of non-ionic plastics: evolution of charge density with contact time. For all of the polymers explored here (acetal, PTFE, PVC, PDMS, nylon), charging magnitude initially increased, reached a maximum value, and then dropped. Error bars are the $95 \%$ confidence limit of the mean charge value, ${ }^{32}$ and are obtained from the analysis of three independently prepared and analysed samples.

system removes complexity introduced by factors such as differences in surface adhesion, hardness and work function. ${ }^{5}$

\section{Results and discussion}

Surface mechanical properties are known to affect material transfer, 5,26 and hence the charge exchange between two polymers. Further, material-specific ion stabilities ${ }^{22}$ contribute to define the mosaic pattern of positive and negative domains that sum up to give a tribocharged sample its net (measurable) charge. Faraday pail charge measurements of identical materials are arguably the simplest laboratory model for the kinetic analysis of charge build-up. Acetal, polytetrafluoroethylene (PTFE), polyvinyl chloride (PVC), polydimethylsiloxane (PDMS) and nylon samples were tribocharged against surfaces of the same material while the contact time for different experiments was systematically increased (Fig. 1). To a first approximation, based on empirical data from readily available triboelectric series, 33,34 contact between identical materials should result in zero charge density. A given material is characterised by a unique macroscopic work function, hence the lack of an "electronic" driving force makes it reasonable to expect a nil charge developing between identical polymers. This has been reported not to be the case, ${ }^{35-37}$ and is further validated by the data shown in Fig. 1. Pressing two identical polymers together, followed by separation, causes transfer of charged material. ${ }^{18,}$ 20, 22 A small and unavoidable asymmetry in the applied mechanical force, and/or a small difference in hardness and adhesion (Fig. S1, ESI + ), 5 leads to triboelectrification even of identical materials. ${ }^{38}$ The charge measured on each sample is the arithmetic sum of positive and negative charges carried by atoms or molecules being transferred, and very surprisingly for all the non-ionic dielectrics studied here (acetal, PTFE, PVC, PDMS, and nylon) this value reaches a maximum after a finite, and relatively short, charging time. Taking for example the case of PTFE, at its charge peak point one sample bears a net positive charge $\left(2.9 \mathrm{nC} / \mathrm{cm}^{2}\right.$, Fig. 1$)$ while the other sample is negative $\left(-3.2 \mathrm{nC} / \mathrm{cm}^{2}\right)$. In the discussion that follows we specifically focus on the negatively charged sample, as this is expected to mediate the reduction of larger amounts of reducible species ${ }^{39}$ and find potential applications in electrochemistry and catalysis. ${ }^{22,30}$ Charge versus time curves for the positive sample are in Fig. S2, ESIt. Most importantly, for all the plastic materials in Fig. 1, charge densities decrease after having reached a peak value. 


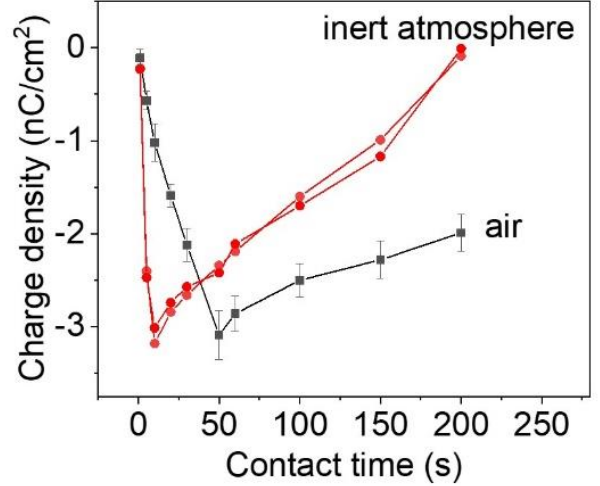

Fig. 2. Faraday pail data showing charge density versus contact time for the negatively charge sample of a PTFE-PTFE dynamic contact. Experiments were performed either inside a glove box (red trace) or in air (black trace). Error bars are the 95\% confidence limit of the mean of three independent experiments.

One first possible explanation for the charge dropping past a peak point is the consumption of adsorbed water. One of the proposed mechanisms - but not yet unambiguously validated ${ }^{30}$ - by which a non-ionic polymer gains static charges upon contact is the asymmetric movement of hydroxide ions. ${ }^{31,40} \mathrm{~A}$ thin water layer ${ }^{41}$ should therefore facilitate charging of a dynamic polymer contact. It is then possible that when water is depleted, further charging is impeded. To test this hypothesis we carried out contact charging experiment inside a glove box with only ppm levels of water. Data in Fig. 2 show static charging of PTFE samples reaching maximum value at shorter charging times inside a glove box compared to data in ambient air. Therefore, consumption of adsorbed water (possibly via a limited availability of hydroxide ions ${ }^{40}$ ) is probably a factor impeding further tribocharging. In fact, the surface of nylon is significantly more hydrophilic ${ }^{42}$ than that of PTFE, ${ }^{43}$ and the onset of the charge peak in nylon is the slowest we have observed (Fig. 1). Water tendency to strongly adsorb on thermoplastics materials is well-known. ${ }^{25}$ For instance, water adsorption accounts for lengthy drying procedures during the processing of thermoplastics. Surface water is also known to play a crucial role in the electrification of dielectrics, ${ }^{44}$ suggesting that our experiments may be affected by a supply of water trapped by the polymer. Control experiments performed by pre-heating PTFE samples at $110^{\circ} \mathrm{C}$ inside a glove box led to tribocharging versus time curves comparable to those recorded under inert atmosphere at room temperature (Fig. S3, ESI ${ }^{+}$). This finding suggests that a DCM wash of the sample is an adequate procedure in terms of removing strongly adsorbed water from hydrophobic surfaces. ${ }^{41}$ However we note that water adsorption alone cannot explain trends in charging magnitude: hydrophilic nylon charges very little. We also considered the possibility of glass transition affecting material transfer hence electrification. ${ }^{5}$ It is possible for the glass transition temperature to be reached at localised spots, hence triggering a change in the charging slope in response to changes to the mechanical properties of plastics, which could affect material transfer between the two surfaces. However, partially against this hypothesis is the fact that the glass transition temperature for nylon, the material with the slowest time to reach a maximum charge value, is as low as $40-50{ }^{\circ} \mathrm{C}, 45$ for instance is significantly lower than that of PTFE $\left(127^{\circ} \mathrm{C}\right) .4^{46}$

The change in the sign of the charging slope around a specific point is associated with a change in surface morphology. AFM data in Fig. 3 show that the surface roughness of PTFE is at its lowest at the charge density peak. Surface roughness increases again past this point. In more detail, two PTFE samples, labelled as PTFE 1 and PTFE 2, were washed with water, ethanol and DCM to remove charges, ${ }^{20}$ and then tribocharged (i.e., PTFE 1 was rubbed against PTFE 2). One sample gains a net-positive charge, while the other gains a net-negative charge of similar magnitude (within 91\%). AFM topography images in Fig. 3a show both PTFE samples becoming significantly smoother at a contact time of $50 \mathrm{~s}$, which is roughly equivalent to the time required for tribocharges to reach their maximum value (Faraday pail data, Fig. 1), and then roughen again past this point. Despite both samples becoming initially smoother, one consistently appears to be rougher than the other, and in the case of PTFE, the sample showing the largest initial change in surface topography is PTFE 1 (the sample with a net-positive charge), while the surface with the least topographical change gains a net-negative charge (Fig. 3a). To reconcile the observed topography changes (Fig. 3a and $3 \mathrm{~b}$ ) with the non-monotonic change in surface charging in Fig. 1, we tentatively propose a material transfer mechanism depicted in Fig. 4. The electrification of identical materials involves the movement of ionic polymer fragments, 7,22 and the partition of these fragments between the two PTFE surfaces is clearly asymmetric (a)

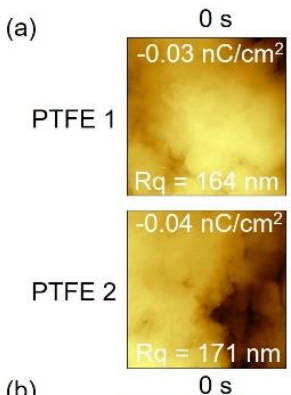

(b)

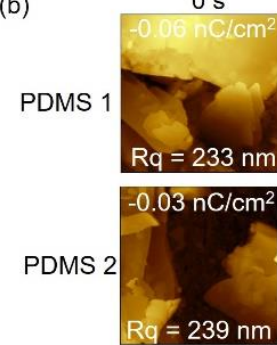

$50 \mathrm{~s}$
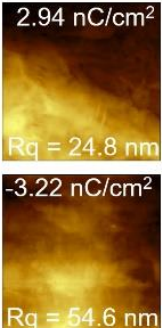

$20 \mathrm{~s}$
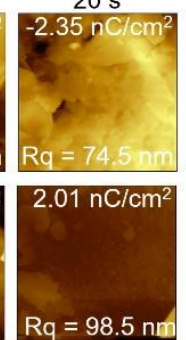

$100 \mathrm{~s}$

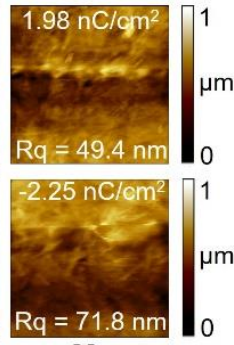

$30 \mathrm{~s}$

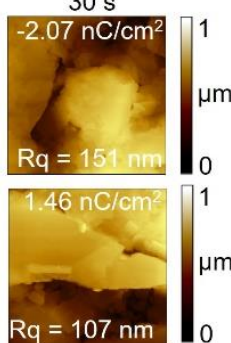

Fig. 3. AFM topography images of PTFE and PDMS samples involved in sliding PTFE-PTFE and PDMS-PDMS contacts. The charge contact time is 0,50 and $100 \mathrm{~s}$ for PTFE (a), and 0, 20 and $30 \mathrm{~s}$ for PDMS (b). Values of surface roughness (reported as root mean square surface roughness, $\mathrm{Rq}$ ) and of charge density (Faraday pail) are indicated in figure. Imaged areas are $10 \times 10$ $\mu \mathrm{m}$ for all samples. 


\section{ARTICLE}

๑) cationic fragment

anionic fragment

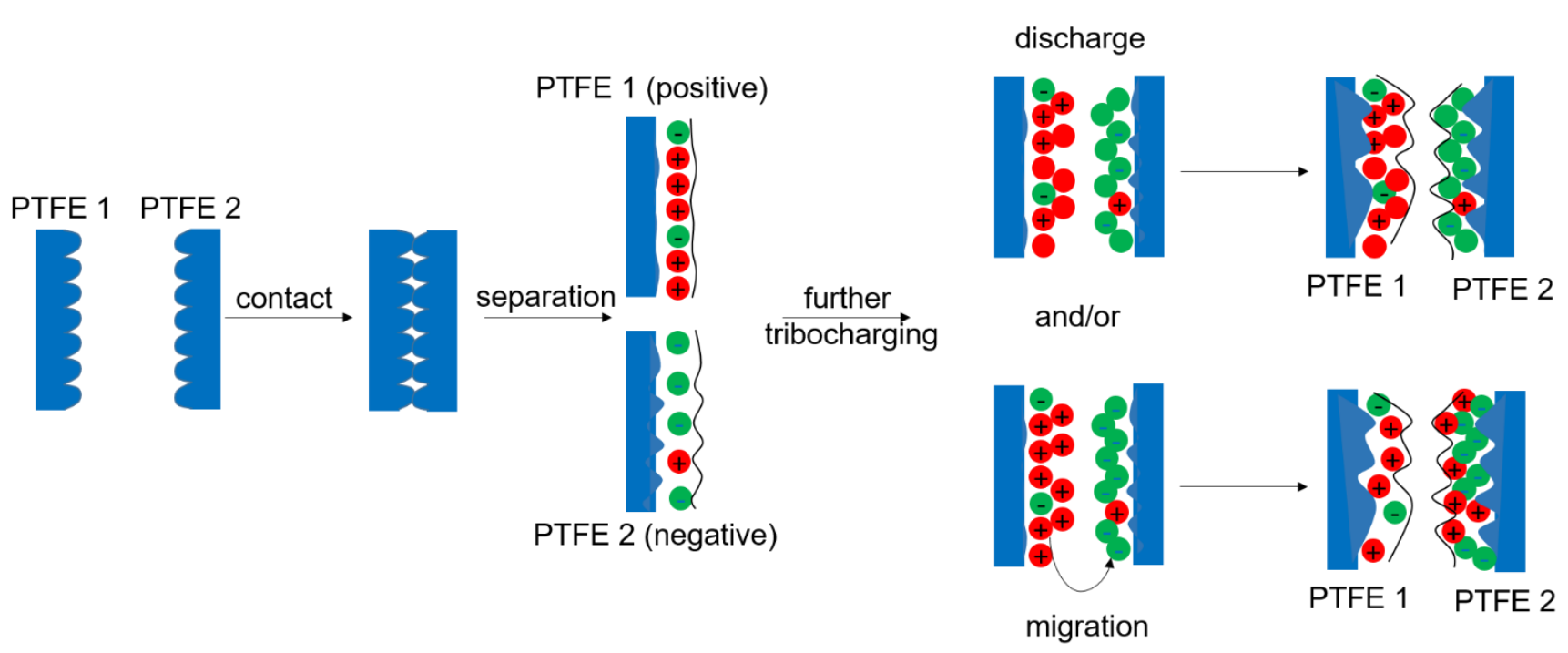

Fig. 4. Schematic depiction of the charge evolution on PTFE samples continuously rubbed against each other (see the time-resolved charge and topography data in Fig. 3). Upon contact and separation, one PTFE sample becomes positive and the other negative. The charge density of both surfaces reaches a maximum value at the certain time (ca. $50 \mathrm{~s}$ for PTFE, a situation depicted after the "separation" step) and then decreases upon further tribocharging. The cause of this charge drop is tentatively ascribed to either surface discharge by dielectric breakdown at sufficiently large surface charge densities (hence fields), or to migration and asymmetric segregation of charged polymer fragments. Optimized geometries as Gaussian Archive entries, and 3D chemical structures of the ionic fragments depicted here simply as plus and minus symbols, are found in the ESIt. For clarity only one migration direction (cations towards anions) is depicted in figure.

(based on charge and topography differences, Fig. 3). In order to appear macroscopically positive, one surface (here PTFE 1) must have gathered an excess of unstable cationic fragments, many of whom probably drop to neutral rapidly, and is thus smoother. The other surface, PTFE 2, need only to gain a small excess of relatively stable anionic fragments to reach a netnegative charge, and appears to be rougher (central block in Fig. 4).

A similar trend was found for PDMS samples, which unlike PTFE generate stable cations and unstable anions (see computed IE and EA values in Fig. 5). AFM topography images in Fig. 3b show that both PDMS surfaces become smoother after a tribocharging time of $20 \mathrm{~s}$ (the charge peak time for PDMS, Fig. 1). A net-negative charge on PDMS is measured for the sample with the greater topography change (PDMS 1 ). In other words, as for PDMS, the smoother surface is the one bearing a large number of unstable fragments (in the case of PDMS anionic fragments). To clarify further on the links between charging and roughness, we performed contact charging experiments on PTFE samples of different initial roughness (Fig. S4, ESI+). The charge density peak magnitude is smaller on smooth PTFE than it is on rougher samples ( $12 \%$ smaller). The time required to reach this peak is also longer on smooth samples (ca. $40 \%$ longer). We postulate a major factor behind these differences is that a rough surface is more likely to transfer material upon friction.

The AFM results from PTFE and PDMS dynamic contacts provide indirect evidence that ionic fragments transfer upon contact charging between identical materials and also outline a possible path to help predicting surface charge polarities from topographical changes. ${ }^{47}$ In order to remove the variation of the surface properties as a function of radius from the centre of rotation, only the centre of each sample is measured by AFM. For explanation for the observed drop in charge density after the peak point (right portion of Fig. 4) we put forward two mechanisms. Firstly, it is possible to envision the electrostatically driven migration of anionic and cationic fragments. For instance in the PTFE system, cationic fragments from PTFE 1 will migrate towards PTFE 2 (or vice versa, PTFE 2 anions in the opposite direction; the net charging effect remaining the same). Secondly, since the charge drop begins only beyond a certain charge magnitude (the peak point), it is possible that unstable surface ions will begin to discharge by dielectric breakdown but only when a certain surface excess of unstable ions is reached (the large charge density on the surface, leads to a large electric filed outside it). This will result in the rate of surface charging beginning to drop behind the rate of material transfer, hence explaining the further increase in 
roughness when the charge starts dropping beyond peak point (Fig. 3).

Unfortunately we do not have a model that could help predicting, simply based on materials electron affinities and ionization energies, when in relative terms a material reaches its charge maxima. There appears to be no correlation between the stability of charged polymer ions and tribocharging peak time. For example, both nylon and PTFE are slow in reaching the curve maxima, but the two materials share little similarities when it comes to ionization energies (IE) and electron affinities

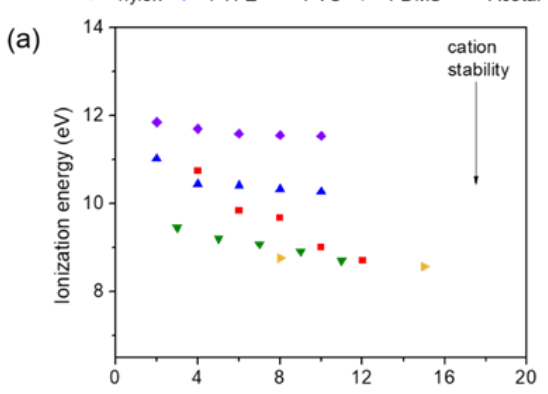

(b)
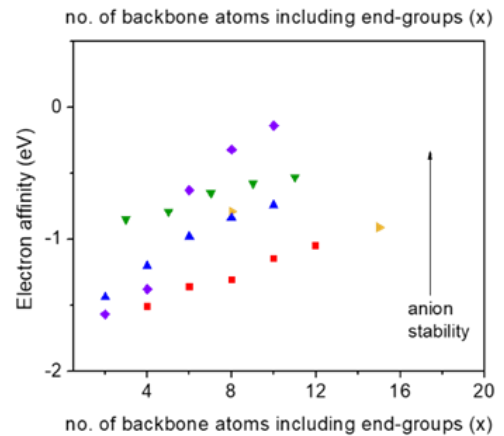

Fig. 5. (a) Computed gas-phase $298 \mathrm{~K}$ ionization energies (IE) and (b) electron affinities (EA) for oligomers of acetal, PTFE, PVC, nylon and PDMS as a function of the number (no.) of backbone atoms including end-groups (Table S1). Values for Acetal are computed in this work; all others taken from Ref 13 For all structures, see Fig. S5, ESIt.

(EA) (Fig. 5). Also, the short peaking times measured for acetal, PVC and PDMS, again are hard to reconcile with similarities in EA and IE computed values. Further studies on this are required, but we believe that differences in material transfer rates and water adsorption, rather than the relative stability of cationic and anionic fragments, govern the onset of the charge peak point. Mechanochemistry aspects of the tribocharging process have been discussed extensively elsewhere. ${ }^{48-51}$

\section{Experimental}

Materials. Redistilled solvents and Milli- $\mathrm{Q}^{\mathrm{TM}}$ water $(>18 \mathrm{M} \Omega \mathrm{cm})$ were used for substrate cleaning and to prepare electrolytic solutions. Samples of acetal (polyoxymethylene, 514-338, RS Components Australia ${ }^{\circledR}$ ), PTFE (polytetrafluoroethylene, 680640 , RS Components Australia ${ }^{\circledR}$ ), PDMS (polydimethylsiloxane, molecular weight of $500 \mathrm{kDa}, 840-5541$, RS Components
Australia $^{\circledR}$ ) and PVC (polyvinyl chloride, 321-2105, RS Components Australia ${ }^{\circledR}$ ) were $1 \times 1 \mathrm{~cm}$ in size and approximately $0.3 \mathrm{~cm}$ in thickness. Samples of nylon (nylon-66, 514-607, RS Components Australia ${ }^{\circledR}$ ) were disks of $1 \mathrm{~cm}$ radius and of a thickness of approximately $0.3 \mathrm{~cm}$.

Charge Measurements. Plastic samples were washed with Milli$\mathrm{Q}^{\mathrm{TM}}$ water (>18 $\left.\mathrm{M} \Omega \mathrm{cm}\right)$, rinsed three times with both methanol and DCM, dried under a nitrogen flow, then stored under vacuum before being statically charged. One sample was fixed at the lower end of a rapidly rotating mechanical shaft (ca. 90 rpm) and then pressed against a second sample (of the same material) secured onto a stationary wood surface. Unless otherwise specified, the charging experiments were performed under ambient air with a relative humidity of $25 \%$. To ensure reproducible contact force during the charging procedure a spring (spring constant of $4.8 \mathrm{~N} / \mathrm{m}$ ) was pressed against the backside of the static sample. The duration of the charging procedure was varied between $0 \mathrm{~s}$ and $350 \mathrm{~s}$, after which the sample net charge was immediately measured in a Faraday pail. The Faraday pail was a JCl 140 static monitor coupled to a JCl 147 charge measurement units, purchased and calibrated by DEKRA (Southampton Science Park, Southampton, UK) and operating on the $10^{-9}$ Coulomb scales, respectively. Charging data are reported as charge density (charge-to-geometric area ratios). Experiments and charge measurements under an argon atmosphere were conducted in an Innovative Technology glove box (PL-HE-2GB fitted with a PL-HE-GP1 inert gas purifier), with water and oxygen levels below $1 \mathrm{ppm}$. The 95\% confidence interval of the $R q$ mean value $(x)$ is reported as $x \pm t_{n-1} s / n^{1 / 2}, 60$ where $t_{n-1}$ depends on the number of repeats and was 4.30 , $s$ is the standard deviation, and $\mathrm{n}$ is the number of measurements (here $\mathrm{n}$ was three).

Computational Procedures. All calculations were performed using Gaussian 16 Rev C.0152 at the M06-2X/6-31+G(d) level of theory ${ }^{53}$ for consistency with our earlier study. ${ }^{22}$ Where available, DFT-computed ionization energies (IEs) and electron affinities (EAs) were taken from our previous study. ${ }^{22}$ For the polyacetal oligomers, calculations for each chain length $\mathrm{n}$ were performed using a methyl and a hydroxyl as the two endgroups. Calculations for larger nylon fragments are at this stage computationally not feasible. Geometries, frequencies and energies were obtained at M06-2X/6-31+G(d). For the gas phase IEs and EAs, thermal corrections were calculated at $298.15 \mathrm{~K}$ using the harmonic oscillator rigid rotor approximation; frequencies were scaled. In continuation of our previous study on tribocharging, our acetal systems have been modelled with the M06-2X/6-31+G(d) level of theory using the quantum chemistry package Gaussian 16 Rev C.01. ${ }^{54,} 52$ Thermal corrections were calculated at $298.15 \mathrm{~K}$ with scaling assuming the ideal gas molecular partition functions in conjunction with the rigid rotor harmonic oscillator (RRHO) approximation, whilst solvent effects in water were addressed with the SMD solvation model using default settings. ${ }^{55}$ Anions and cations, all open-shell, were treated with an unrestricted wavefunction. Assuming a methyl and a hydroxyl end-group, geometries for chains of increasing monomer count were constructed in the molecular visualisation package IQmol. Full conformer searching was then performed up to the polyacetal tetramer, with the directed tree search algorithm employed to efficiently search the large conformational space of the longest species considered here, the pentamer. ${ }^{56}$ All final structures were characterised as local minima by way of vibrational analysis. 
The adiabatic electron affinity $(E A)$ and ionisation potential $(I P)$ at $298.15 \mathrm{~K}$ have been calculated as follows,

$$
\begin{gathered}
E A=-\Delta H_{0}(+e), \\
I P=\Delta H_{0}(-e),
\end{gathered}
$$

where the subscript denotes kelvin. The reaction enthalpy $\left(\Delta H_{0}\right)$ is calculated from the heats of formation for the redox couple in the gas phase, assuming the electron convention with Fermi-Dirac statistics (EC-FD) for the electron, which specifies $H_{0}(e)$ as $3.145 \mathrm{~kJ}$ $\mathrm{mol}^{-1}$ at $298.15 \mathrm{~K}$. For compactness, these are given in electron volts.

\section{Conclusions}

In this work we have studied the evolution, with contact time, of the electrification of two identical insulating materials brought in and out of contact. We have shown for the first time that even upon continuous charging the net charge magnitude peaks after an initial increase, and then steadily drops. The transfer of ionic fragments is the main cause of electrification, but asymmetry in the material transfer, dielectric breakdown, fragment migration and adsorbed water appear to be more important than the stability of the surface charges in explaining why charging magnitude versus contact time data do not follow a monotonic curve. The existence of two branches in the charging curve-implying two charging times can yield the same output - is probably one of the reasons why it is generally difficult to predictably obtain a precise charging magnitude. This work extends our understanding and control over static electricity with immediate implications in single-electrode electrochemistry and in aiding the study of electrostatic forces on chemical reactivity. ${ }^{5,30,57}$

\section{Conflicts of interest}

There are no conflicts to declare.

\section{Acknowledgements}

This work was financially supported by the Australian Research Council (DP190100735 (S.C. and N.D.), FT190100148 (S.C.), and FL170100041 (M.L.C)).

\section{Notes and references}

1. J. Zhang, N. Darwish, M. L. Coote and S. Ciampi, Adv. Eng. Mater. 2019, 1901201.

2. K. Zhang and Y. Yang, Nano Res. , 2016, 9, 974-984.

3. K. Zhao, Z. L. Wang and Y. Yang, ACS Nano, 2016, 10, 9044-9052.

4. X. Zhao, B. Chen, G. Wei, J. M. Wu, W. Han and Y. Yang, Adv. Mater. Technol. , 2019, 4, 1800723.

5. J. Zhang, S. Ferrie, S. Zhang, Y. B. Vogel, C. Peiris, N. Darwish and S. Ciampi, ACS Appl. Nano Mater., 2019, 2, 7230-7236.

6. G. Castle, J. Electrost., 1997, 40, 13-20.

7. J. Zhang and S. Ciampi, Aust. J. Chem., 2019, 72, 633-636.
8. Z. L. Wang and A. C. Wang, Mater. Today, 2019, 30, 1369-7021.

9. S. Chuetor, R. Luque, C. Barron, A. Solhy, X. Rouau and A. Barakat, Green Chem., 2015, 17, 926-936.

10. T. Sueto, Y. Ota and K. Nishioka, Sol. Energy, 2013, 97, 414-417.

11. B. Chen, N. Yang, Q. Jiang, W. Chen and Y. Yang, Nano Energy, 2018, 44, 468-475.

12. T. Gao, K. Zhao, X. Liu and Y. Yang, Nano Energy, 2017, 41, 210216.

13. X. Liu, K. Zhao, Z. L. Wang and Y. Yang, Adv. Energy Mater., 2017, 7, 1701629.

14. T. Quan, Z. L. Wang and Y. Yang, ACS Appl. Mater. Interfaces 2016, 8, 19573-19578.

15. T. Quan, Y. Wu and Y. Yang, Nano Res. , 2015, 8, 3272-3280.

16. Y. Su, Y. Yang, X. Zhong, H. Zhang, Z. Wu, Y. Jiang and Z. L. Wang, ACS Appl. Mater. Interfaces 2014, 6, 553-559.

17. Y. Yang, W. Guo, K. C. Pradel, G. Zhu, Y. Zhou, Y. Zhang, Y. Hu, L. Lin and Z. L. Wang, Nano Lett., 2012, 12, 2833-2838.

18. H. Baytekin, A. Patashinski, M. Branicki, B. Baytekin, S. Soh and B. A. Grzybowski, Science, 2011, 333, 308-312.

19. M. Mirkowska, M. Kratzer, C. Teichert and H. Flachberger, Chem. Ing. Tech., 2014, 86, 857-864.

20. T. A. Burgo, T. R. Ducati, K. R. Francisco, K. J. Clinckspoor, F. Galembeck and S. E. Galembeck, Langmuir, 2012, 28, 74077416.

21. K. Brown and G. Tautfest, Rev. Sci. Instrum, 1956, 27, 696-702.

22. J. Zhang, F. Rogers, N. Darwish, V. R. Gonçales, Y. B. Vogel, F. Wang, J. J. Gooding, M. C. Peiris, G. Jia, J.-P. Veder, M. L. Coote and S. Ciampi, J. Am. Chem. Soc., 2019, 141, 5863-5870.

23. S.-H. Shin, Y. E. Bae, H. K. Moon, J. Kim, S.-H. Choi, Y. Kim, H. J. Yoon, M. H. Lee and J. Nah, ACS Nano, 2017, 11, 6131-6138.

24. I. A. Harris, M. X. Lim and H. M. Jaeger, Phys. Rev. Mater., 2019, 3, 085603-085612.

25. T. A. Burgo, F. Galembeck and G. H. Pollack, J. Electrost., 2016, 80, 30-33

26. H. T. Baytekin, B. Baytekin, J. T. Incorvati and B. A. Grzybowski, Angew. Chem., Int. Ed., 2012, 51, 4843-4847.

27. M. Kowacz and G. H. Pollack, J. Phys. Chem. B, 2019, 123, 1100311013.

28. C.-Y. Liu and A. J. Bard, Chem. Phys. Lett., 2010, 485, 231-234.

29. C. Liu and A. J. Bard, J. Am. Chem. Soc., 2009, 131, 6397-6401.

30. C.-Y. Liu and A. J. Bard, Nat. Mater. , 2008, 7, 505-509.

31. J. A. Wiles, M. Fialkowski, M. R. Radowski, G. M. Whitesides and B. A. Grzybowski, J. Phys. Chem. B, 2004, 108, 20296-20302.

32. J. Miller and J. C. Miller, Statistics and chemometrics for analytical chemistry, Pearson education, 2018.

33. D. M. Gooding and G. K. Kaufman, Encycl. Inorg. Bioinorg. Chem. John Wiley \& Sons, Ltd, , 2014, PP. 1-9.

34. H. Zou, Y. Zhang, L. Guo, P. Wang, X. He, G. Dai, H. Zheng, C. Chen, A. C. Wang and C. Xu, Nat. Commun., 2019, 10, 1427-1435.

35. M. M. Apodaca, P. J. Wesson, K. J. Bishop, M. A. Ratner and B. A. Grzybowski, Angew. Chem., Int. Ed., 2010, 49, 946-949.

36. T. Shinbrot, T. Komatsu and Q. Zhao, EPL 2008, 83, 24004-24009.

37. R. Pham, R. C. Virnelson, R. M. Sankaran and D. J. Lacks, J. Electrost., 2011, 69, 456-460.

38. M. W. Williams, AIP Adv., 2012, 2, 010701

39. Both pieces should have negative and positive charges, but negative one should have more negative charges than positive one.

40. L. S. McCarty and G. M. Whitesides, Angew. Chem., Int. Ed., 2008, 47, 2188-2207. 
41. M. James, T. A. Darwish, S. Ciampi, S. O. Sylvester, Z. Zhang, A. Ng, J. J. Gooding and T. L. Hanley, Soft Matter, 2011, 7, 53095318.

42. J. Du, L. Zhang, J. Dong, Y. Li, C. Xu and W. Gao, J. Eng. Fibers Fabr., 2016, 11, 31-37.

43. A. Hausmann, P. Sanciolo, T. Vasiljevic, M. Weeks, K. Schroën, S. Gray and M. Duke, J. Membr. Sci., 2013, 442, 149-159.

44. R. F. Gouveia and F. Galembeck, J. Am. Chem. Soc., 2009, 131, 11381-11386.

45. R. Greco and L. Nicolais, Polymer, 1976, 17, 1049-1053.

46. Y. Araki, J. Appl. Polym. Sci., 1965, 9, 421-427.

47. For the homo-systems of our experiments, it was to some degree possible to infer charging signs from topographical data alone. For materials with unstable cations and relatively stable anions, such as PDMS, a rougher surface was often indicative of a net positive charge carried by the sample, while a smoother surface of a negative-sign charging. When dealing with materials with unstable anions and relatively stable cations, such as PTFE, the rougher surface was generally bearing a negative charge while the smoother was positive.

48. F. Galembeck, T. A. Burgo, L. B. Balestrin, R. F. Gouveia, C. A. Silva and A. Galembeck, RSC Adv., 2014, 4, 64280-64298.

49. L. B. da Silveira Balestrin, D. Del Duque, D. S. da Silva and F. Galembeck, Faraday Discuss., 2014, 170, 369-383.

50. T. A. Burgo, C. A. Silva, L. B. Balestrin and F. Galembeck, Sci. Rep., 2013, 3, 2384-2391.

51. L. P. Santos, D. S. da Silva, B. C. Batista, K. S. Moreira, T. A. Burgo and F. Galembeck, Polymer, 2019, 171, 173-179.

52. M. Frisch, G. Trucks, H. Schlegel, G. Scuseria, M. Robb, J. Cheeseman, G. Scalmani, V. Barone, G. Petersson and $\mathrm{H}$. Nakatsuji, Gaussian 16, revision A.03; Gaussian Inc.: Wallingford, CT, 2016.

53. Y. Zhao and D. G. Truhlar, Theor. Chem. Acc. , 2008, 120, 215241.

54. Y. Zhao and D. G. Truhlar, Theor. Chem. Acc., 2008, 120, 215-241.

55. A. V. Marenich, C. J. Cramer and D. G. Truhlar, J. Phys. Chem. B, 2009, 113, 6378-6396.

56. E. I. Izgorodina, C. Yeh Lin and M. L. Coote, Phys. Chem. Chem. Phys., 2007, 9, 2507-2516.

57. S. Shaik, D. Mandal and R. Ramanan, Nat. Chem., 2016, 8, 10911098. 
TOC. Contact electrification: Irreproducibility of triboelectric charging magnitudes. Using Faraday pail measurements we show that a monotonous charging slope holds only left or right of a material-specific charge-peak point.

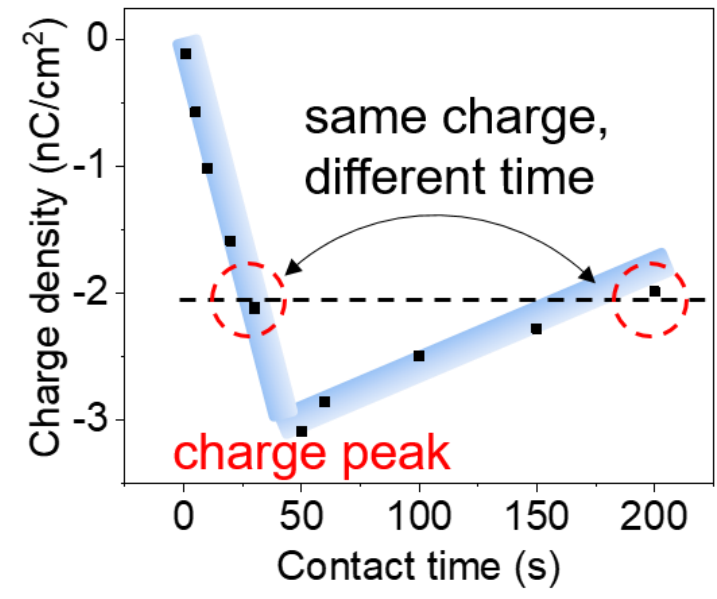

